\title{
Impedance-based sensor for potassium ions
}

\author{
C. Day ${ }^{\mathrm{a}}$, S. Søpstad ${ }^{\mathrm{b}, \mathrm{c}}$, H. Ma ${ }^{\mathrm{d}}$, C. Jiang ${ }^{\mathrm{d}}$, A. Nathan ${ }^{\mathrm{d}}$, S.R. Elliott ${ }^{\mathrm{a}}$, F.E. Karet Frankl ${ }^{\mathrm{e}}$, T. Hutter ${ }^{\mathrm{a}, *}$ \\ ${ }^{a}$ Department of Chemistry, University of Cambridge, Lensfield Road, Cambridge CB2 1EW, United Kingdom \\ ${ }^{b}$ Institute for Microsystems, University College of Southeast Norway, N-3184 Borre, Norway \\ ${ }^{c}$ Zimmer \& Peacock AS, N-3183 Horten, Norway \\ ${ }^{d}$ Electrical Engineering Division, Engineering Department, University of Cambridge, 9 JJ Thomson Avenue, Cambridge \\ CB3 OFA, United Kingdom \\ ${ }^{e}$ Cambridge Institute for Medical Research, Cambridge Biomedical Campus, Cambridge CB2 0XY, United Kingdom
}

\begin{abstract}
A conductometric sensor for potassium ions in solution is presented. Interdigitated, planar gold electrodes were coated with a potassium-selective polymer membrane composed of a poly(vinyl chloride) matrix with about $65 \mathrm{wt} \%$ of plasticiser and $2-5 \mathrm{wt} \%$ of a potassium-selective ionophore. The impedance of the membrane was measured, using the electrodes as a transducer, and related to the concentration of potassium in a sample solution in contact with the membrane. Sensitivity was optimised by varying the sensor components, and selectivity for potassium over sodium was also shown. The resulting devices are compact, miniature, robust sensors which, by means of impedance measurements, eliminate the need for a reference electrode. The sensor was tested for potassium concentration changes of $2 \mathrm{mM}$ across the clinically relevant range of 2.7 - $18.7 \mathrm{mM}$.
\end{abstract}

Keywords: potassium, impedance spectroscopy, conductometric sensor, ion-selective membrane, blood analysis, portable sensor

\section{Introduction}

Detection of potassium-ion concentration in solution is essential for multiple applications, the main ones being soil analysis for crop growth [1, 2], and clinical analysis of blood and other biological fluids [3, 4]. Almost all such sensor devices are based on an ionophore, usually valinomycin, embedded in a plasticised polymer membrane matrix, often poly(vinyl chloride) (PVC). These ion-selective membranes have been widely researched and developed since their introduction in the 1970s [5-7]. Multiple transduction methods have been investigated, principally ion-selective electrodes (ISEs), optodes, and ion-selective field-effect transistors (ISFETs). Mikhelson et al provide an overview [8].

Most commonly employed are ISEs; these are present in traditional off-line methods of soil-sample potassium testing [1], as well as more modern in situ measurement devices [2]. Similarly, ISEs are employed in almost all commercial, clinical blood analysers used in hospitals [9]. Traditional ISEs in larger machines have an internal filling solution, which creates a limit on miniaturisation and durability [10]. However, the demand for miniature sensors for in situ and point-of-care monitoring has increased significantly, and recently, all-solid-state ISEs have been developed which are miniaturisable [4, 10-12]. The requirement for a reference electrode and for frequent calibration, however, remains a drawback of ISEs [10].

Recently, optical methods for potassium detection have become more prevalent, both for crop growth [13] and in blood analyses $[14,15]$. However, spectrophotometers remain relatively large, making miniaturisation

\footnotetext{
* Corresponding author

Email addresses: jcd62@cam.ac.uk (C. Day), sindre@zimmerpeacock.com (S. Søpstad), hm446@cam.ac.uk (H. Ma), cj360@cam.ac.uk (C. Jiang), an299@eng.cam.ac.uk (A. Nathan), sre1@cam.ac.uk (S.R. Elliott), fek1000@cam.ac.uk (F.E. Karet Frankl), tf269@cam.ac.uk (T. Hutter)
} 
difficult. In addition, a review of optodes for clinical use concluded that coupled pH measurements would always be required, which increases complexity [3].

Less widely researched are conductometric sensors. Ion-selective conductometric microsensors (ISCOMs) were developed for potassium and calcium in the 1990s [16-18], but little further work appears to have been done on them. However, these sensors appear promising: they carry the same advantages as solid-state ISEs, in that they are all-solid-state and can be miniaturised. They also have the advantage over potentiometric measurement, in that neither frequent calibration nor a reference electrode is required: a simple conductance measurement of the medium between two electrodes provides the reading.

In addition, there does not currently exist a hand-held blood potassium sensor that is licensed for clinical use; patients must have their blood potassium measured on a large blood analyser in hospital. Most patients who require blood-potassium monitoring are those with kidney disease, who comprise an estimated $6-7 \%$ of the UK population [19], so this leads to a significant use of hospital time and resources [20]. The normal range for potassium concentration in blood is $3.5-5.0 \mathrm{mM}$; in patients with hyperkalaemia (high blood potassium) the concentration can be as high as $10 \mathrm{mM}$ and in patients with hypokalaemia (low blood potassium) it can be as low as $1.5 \mathrm{mM}$. Both can occur in kidney patients, and concentrations below 2.5 or above 6.5 may be dangerous and may require emergency hospital treatment. Both are associated with cardiac rhythm abnormalities, muscle dysfunction and sudden death [21]. For patients with more severe kidney disease, it is possible for potassium levels to become dangerously high between hospital visits which, if unnoticed, can cause severe health problems and even death. At-home measurement would allow patients to monitor their blood potassium and act accordingly, improving patient safety and reducing healthcare costs.

Therefore, the aim of this work was to take steps towards developing a conductometric sensor capable of determining the potassium concentration in human blood. Little recent work has been done on sensors of this type; Shvavev et al developed a conductometric sensor based on impedance measurements [22], but this used very thick ion-selective membranes (20 - $250 \mu \mathrm{m}$ compared to $<1 \mu \mathrm{m}$ in most other works) and consequently sensitivity was low. Braiek et al also worked on a conductometric sensor, based on conductance measurements, recently [23]; however, the blood to be measured was diluted before use by $600 \mathrm{mM}$ sucrose solution in a 1:30 ratio, making the sensor operation relatively complex.

To that end, a sensor consisting of planar gold electrodes coated in a potassium-selective membrane was developed. The membrane composition used was similar to that which has been optimised for ISEs, with a 1:2 ratio of PVC : plasticiser [7]. However, no specific optimisation for conductometric sensors appears to have been been done previously; in this work, the membrane composition was varied and three different ionophores were tested. In addition, the effect of pre-soaking the sensor in concentrated ionic solutions was investigated. This method has been used in previous works, but an explanation of its use was not given; eliminating this step would further simplify the sensing process [17, 23].

\section{Materials and Methods}

\subsection{Reagents}

Valinomycin, potassium ionophore II, potassium ionophore III (see Supplementary Information for details), bis(2-ethylhexyl) sebacate (97\%, Selectophore grade), poly(vinyl chloride) high molecular weight, potassium chloride (99\%, ACS reagent grade), sodium chloride (99\%, ACS reagent grade) and phosphatebuffered saline tablets were purchased from Sigma-Aldrich. Tetrahydrofuran (HPLC grade) was purchased from Fischer Scientific.

\subsection{Electrode design}

Pairs of interdigitated gold electrodes were fabricated in-house on a glass substrate, including a $20 \mathrm{~nm}$ thick titanium layer to improve the adhesion of the $80 \mathrm{~nm}$ thick gold layer to the glass. They were patterned using a lift-off process. Four different arrangements of electrodes were produced, with either a $1 \mathrm{~mm}^{2}$ or $4 \mathrm{~mm}^{2}$ sensitive area, and either $20 \mu \mathrm{m}$ or $40 \mu \mathrm{m}$ wide fingers. The gaps between the fingers were the same width as the fingers themselves. Figure 1a shows a glass wafer containing four electrode pairs, and 
microscope images of the active area can be seen in Figure 1b and in the Supplementary Information [Figure $\mathrm{S} 1]$.

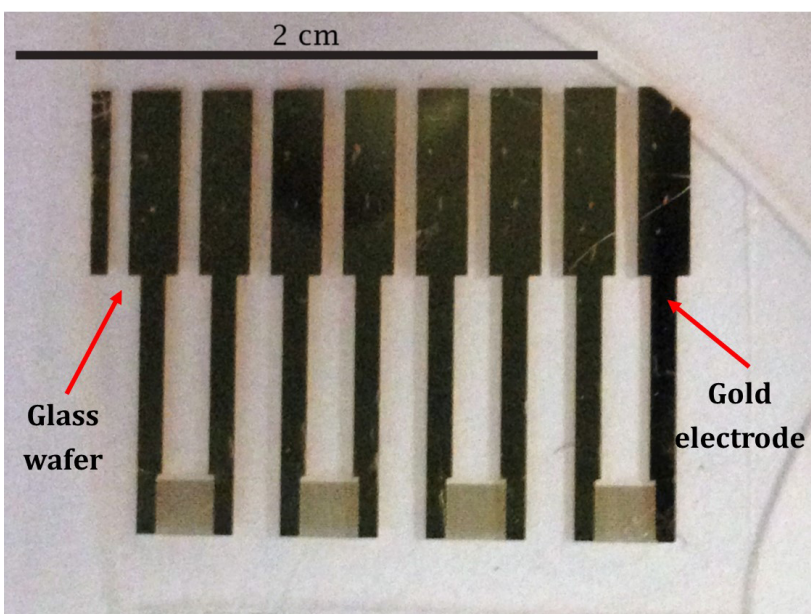

(a) Four electrode pairs, each with $4 \mathrm{~mm}^{2}$ active area.

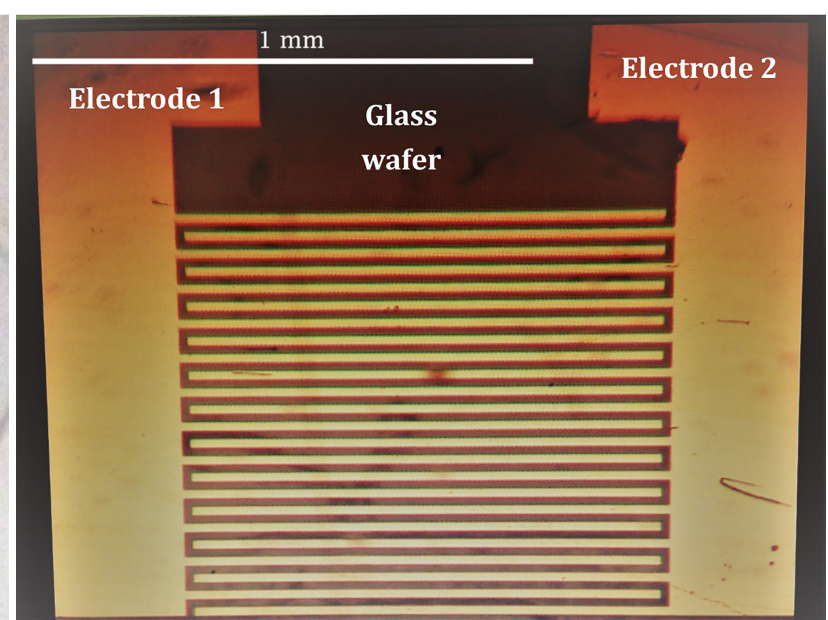

(b) Microscope image of $20 \mu \mathrm{m}$ wide interdigitated fingers.

Figure 1: Interdigitated gold electrode pairs on a glass substrate.

\subsection{Membrane preparation}

Membranes of various compositions were prepared, containing 0 - 5 wt\% ionophore, 31 - 34 wt\% poly(vinyl chloride) (PVC) and 64 - $66 \mathrm{wt} \%$ of the plasticiser bis(2-ethylhexyl) sebacate (DOS) of various dilutions in tetrahydrofuran $(\mathrm{THF})$. For example, the preparation method for a 2 wt\% valinomycin membrane solution was as follows:

PVC $(0.16 \mathrm{~g})$ was stirred in THF $(6 \mathrm{~mL})$, covered to prevent evaporation, for approximately 30 minutes until dissolved. Valinomycin $(0.01 \mathrm{~g})$ and DOS $(0.33 \mathrm{~g})$ were also dissolved in THF $(4 \mathrm{~mL})$ and this solution added to the PVC solution. The mixture was stirred for a further 30 minutes to ensure an even distribution of valinomycin.

Three different potassium ionophores were investigated in this work, to determine which shows the best response when employed in a conductometric sensor architecture. Their molecular structures are given in the Supplementary Information [Figure S2]. Valinomycin is the most commonly used for $\mathrm{K}^{+}$sensing applications.

To apply the membrane to the electrodes, dip-coating using a Langmuir-Blodgett dipper mechanism (NIMA Technology) at a pull speed of $40 \mathrm{~mm} / \mathrm{min}$ was employed and the THF allowed to evaporate, depositing a thin membrane film of approximately $1 \mu \mathrm{m}$ thickness on the surface.

The effect of pre-soaking the membranes was also investigated. Here, the membrane-coated electrodes were submerged in a $0.1 \mathrm{mM}$ solution of either $\mathrm{KCl}$ or $\mathrm{NaCl}$ for at least 24 hours. They were removed from the solution, rinsed briefly with de-ionised water and dried under an air flow before being used for measurements.

\subsection{Impedance measurements}

A home-made mount for the electrodes was built, shown in Figure 2c, with a rubber O-ring to confine the liquid in the cell to only the transducer part of the electrode. During operation, $70 \mu \mathrm{L}$ of phosphate-buffered saline (PBS), containing $137 \mathrm{mM} \mathrm{NaCl}$ and $2.7 \mathrm{mM} \mathrm{KCl}$, was added to the cell and a cover slip placed on top to prevent evaporation. $1 \mathrm{\mu L}$ aliquots of $140 \mathrm{mM} \mathrm{KCl}$ solution were added, each time increasing the concentration of $\mathrm{K}^{+}$in the cell by $2 \mathrm{mM}$ (assuming the volume change is negligible).

Impedance measurements were performed by applying a $500 \mathrm{mV}$ alternating voltage between the two electrodes and scanning through a range of frequencies from $10 \mathrm{~Hz}-1 \mathrm{MHz}$. The DC offset was zero. The 
impedance (magnitude and phase) of the system was measured at intervals of $0.2 \log ($ frequency/Hz). These measurements were carried out on a 1260 Impedance/Gain-Phase Analyser (Solartron). Diagrams of the setup used in this work are shown in Figure 2.

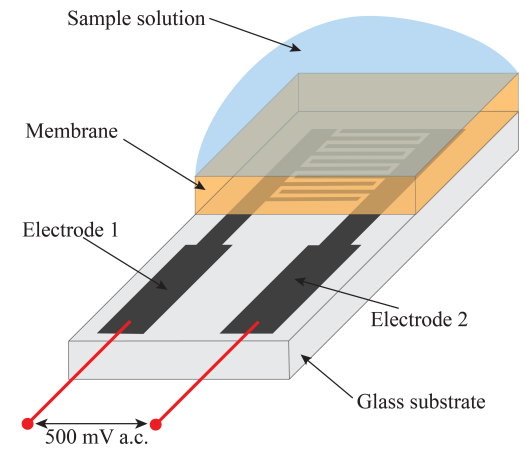

(a) Three-dimensional drawing

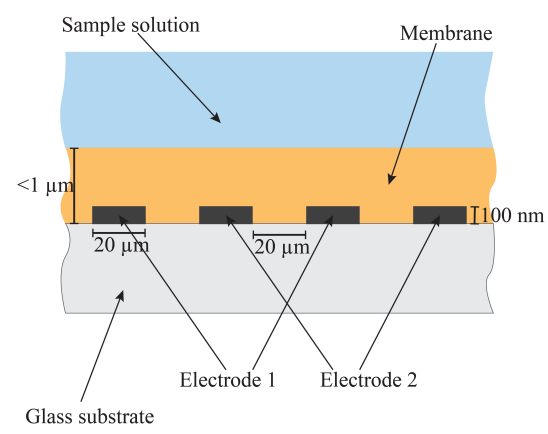

(b) Cross-section

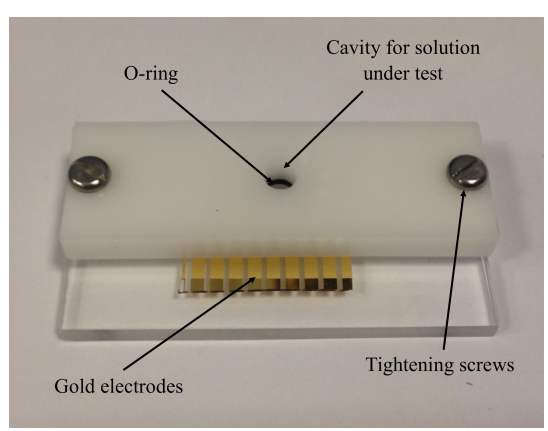

(c) Mount containing an electrode set

Figure 2: Schematics (not to scale) and photograph of the membrane-covered electrodes as set up during measurements. The exposed part of the electrodes is connected by gold pins and wires to the impedance analyser.

\section{Results and Discussion}

\subsection{Raw Data}

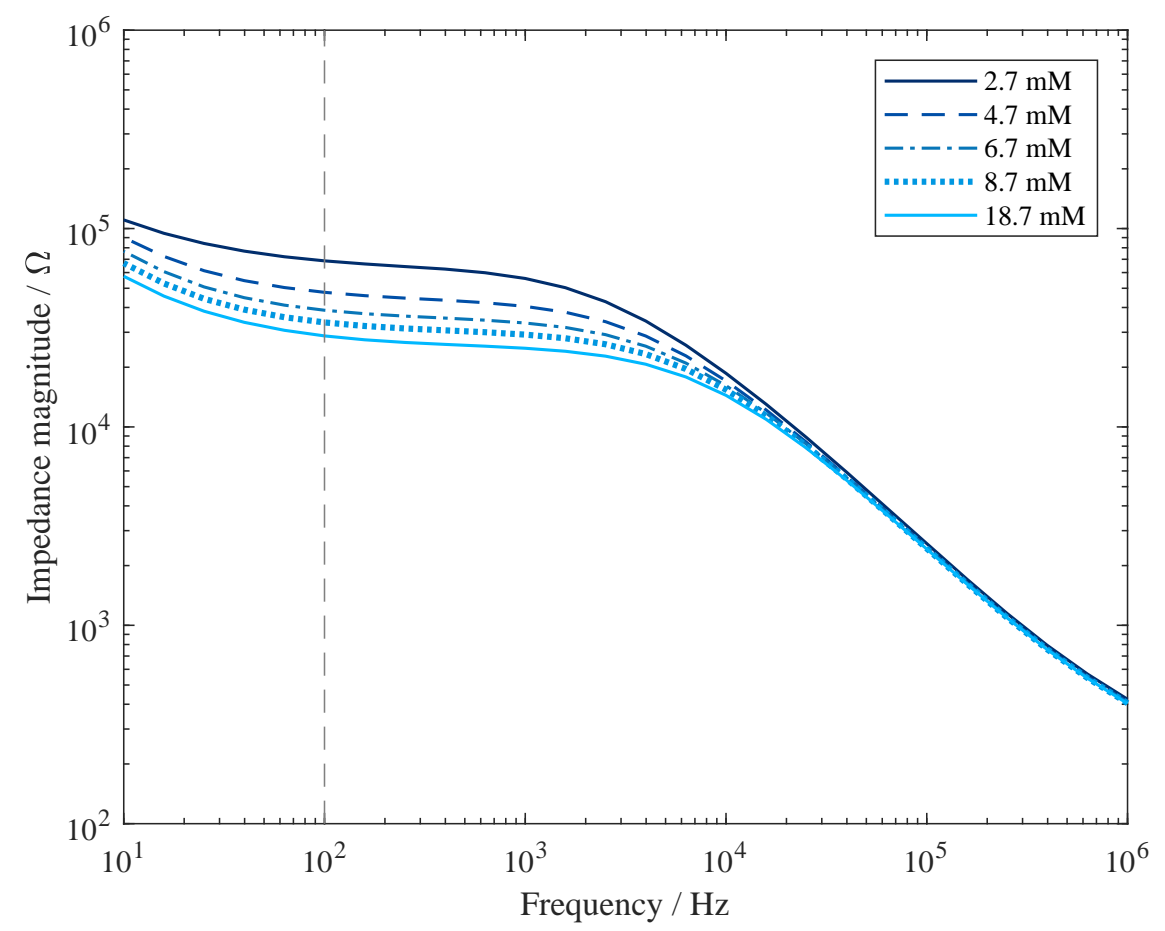

Figure 3: Impedance spectra obtained from sensor S6 (see Table 1) under different $\mathrm{K}^{+}$concentrations.

Typical impedance spectra for the same sensor at different $\mathrm{K}^{+}$concentrations are shown in Figure 3 . At low frequencies, the resistance component of the membrane's impedance is dominant; this is not dependent 
on frequency, hence the plateau in impedance. At higher frequencies, the capacitance contribution to impedance dominates, and hence the impedance decreases as the frequency increases. It can be seen that the largest spread in impedance magnitude between measurements is at about $100 \mathrm{~Hz}$, so this frequency was chosen to present all the data that follow.

\subsection{Curve-fitting analysis}

Figure 4 shows the change in impedance of the sensor over time as aliquots of $\mathrm{KCl}$ were added, as described in Section 2.4. It shows clearly that there is a non-linear decrease in impedance with time, independent of any other factors. A similar time-dependence of the sensor response has previously been observed [22]. An explanation for this is that water is being slowly taken up into the membrane over time, increasing the conductivity. A mechanism for this phenomenon has been proposed, whereby water forms micelles within the hydrophobic membrane structure, allowing ions to move much more freely within these micelles [22]. It can also be seen in Figure 4 that the sensor response appears to be partially reversible.

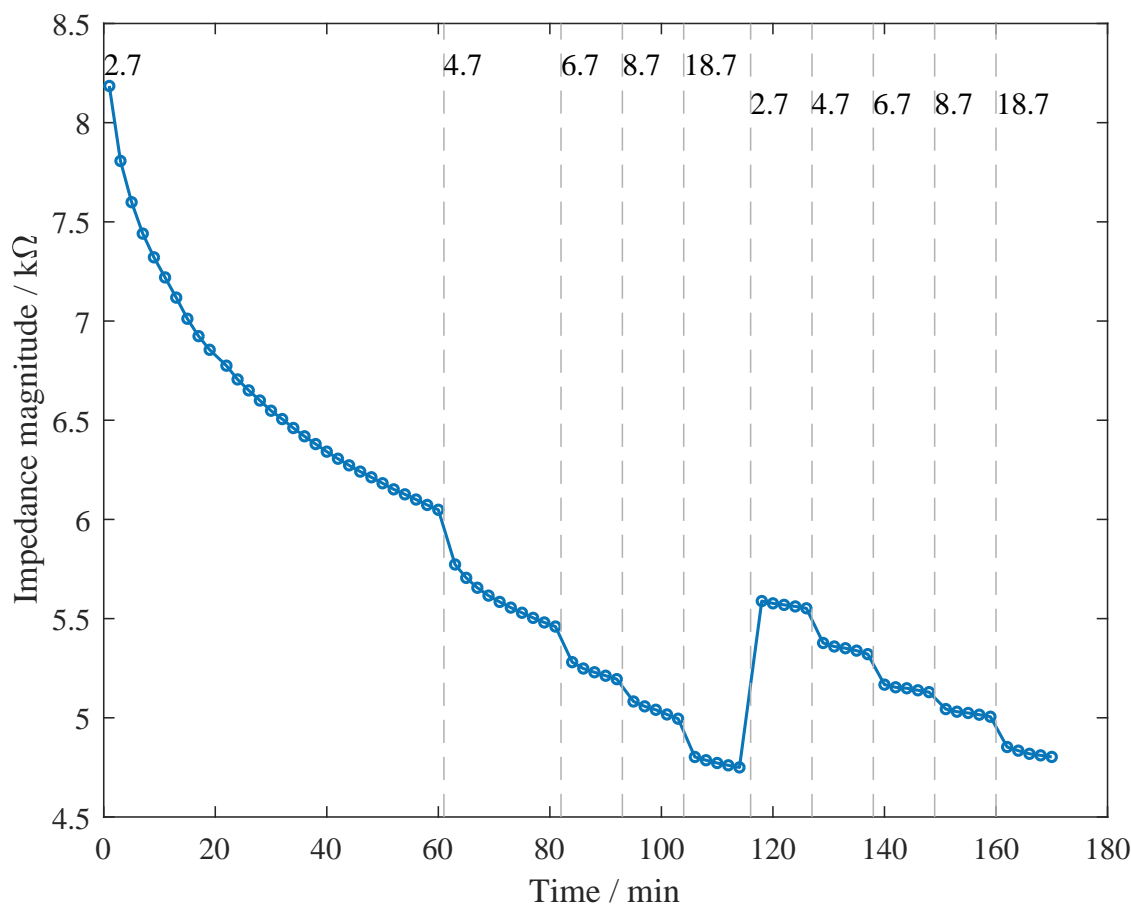

Figure 4: Impedance vs time graph for sensor S5 at $100 \mathrm{~Hz}$. The dashed lines show the times at which the concentration of $\mathrm{K}^{+}$in the sample solution was increased. Concentrations (in $\mathrm{mM}$ ) are indicated at each point. At $\mathrm{t}=118 \mathrm{minutes}$, the sample solution was removed from the cell and replaced with PBS, then the $\mathrm{K}^{+}$concentration was increased stepwise again.

In order to determine the plateau value of impedance, $Z$, curve fitting to the data points for each $\mathrm{K}^{+}$ concentration was performed. An assumption was made that the decay of impedance with time is exponential and an empirical equation of the form $y=Z+b e^{-c t}$ was used. The results of the curve fit are shown in Figure 5, and a table of the coefficients $Z, b$ and $c$ can be found in the Supplementary Information [Table $\mathrm{S} 1]$. The plateau impedance values, $Z$, obtained from the fitted curves were used to create a response vs concentration curve, shown as the inset in Figure 5. A fractional difference method was used, i.e. plotting $\frac{\Delta Z}{Z_{0}}$ against the logarithm of the concentration of $\mathrm{K}^{+}$, where $\Delta Z=\left(Z-Z_{0}\right)$ and $Z_{0}$ is the plateau impedance value in PBS (i.e. $2.7 \mathrm{mM} \mathrm{KCl}$ ). This method is preferred because changes in $Z_{0}$ are eliminated, allowing for better comparison between sensors and removing the effect of the transducer [18]. This plot is a straight line, which agrees with results in [18] and [22]. 


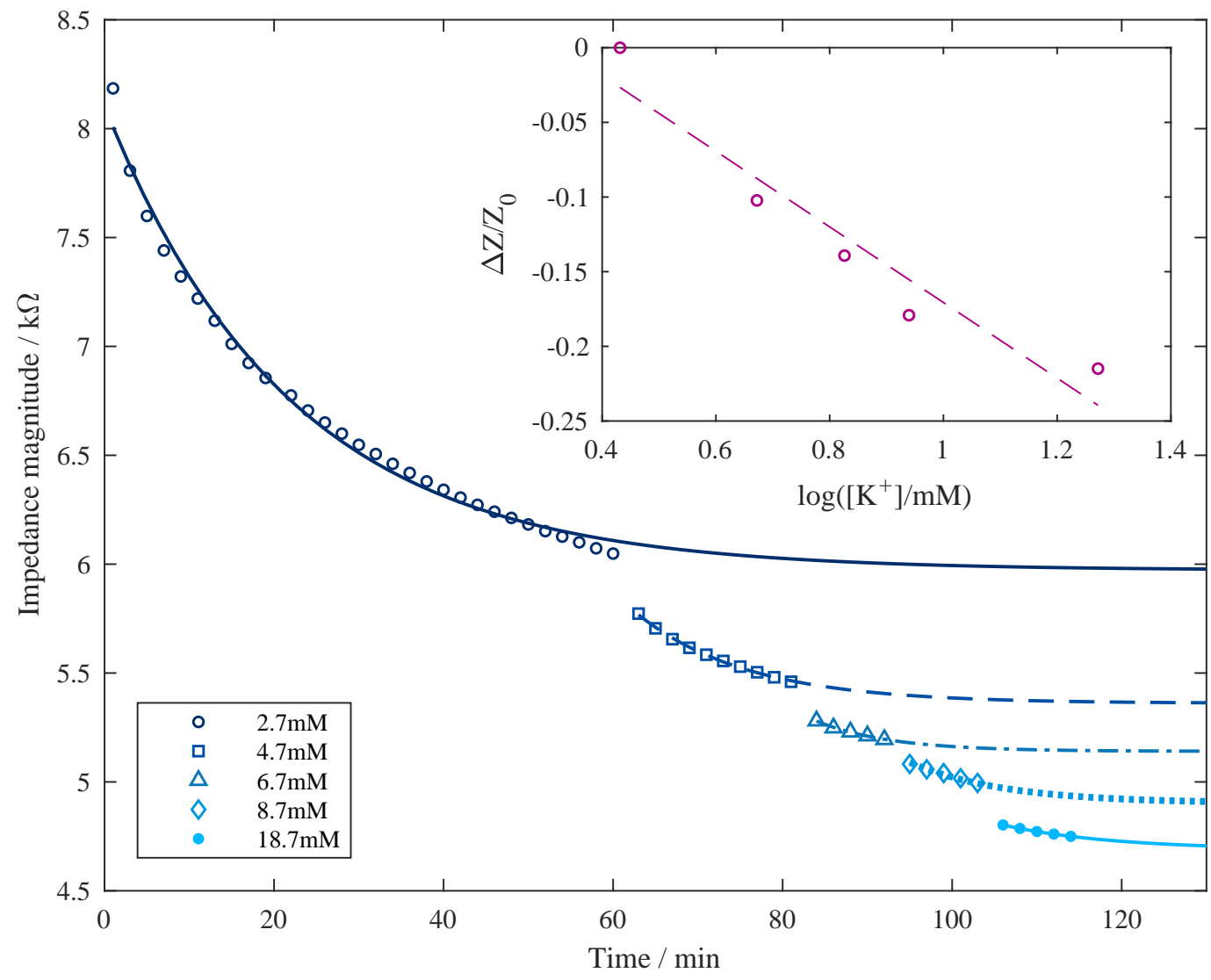

Figure 5: Data points (from Figure 4) and models for each concentration. Inset: calibration curve obtained from the model.

\subsection{Optimisation of sensor design}

Table 1: All tested sensor designs.

\begin{tabular}{ccccccc}
\hline $\begin{array}{c}\text { Sensor } \\
\text { number }\end{array}$ & $\begin{array}{c}\text { Electrode } \\
\text { area } / \mathrm{mm}^{2}\end{array}$ & $\begin{array}{c}\text { Electrode finger } \\
\text { width } / \mu \mathrm{m}\end{array}$ & Ionophore & $\begin{array}{c}\text { Ionophore } \\
\text { conc } / \mathrm{wt} \%\end{array}$ & Pre-soaking & Analyte \\
\hline S1 & 1 & 40 & Valinomycin & 2 & Unsoaked & $\mathrm{KCl}$ \\
S2 & 1 & 40 & Valinomycin & 5 & Unsoaked & $\mathrm{KCl}$ \\
S3 & 4 & 40 & Ionophore II & 5 & Unsoaked & $\mathrm{KCl}$ \\
S4 & 4 & 40 & Ionophore III & 5 & Unsoaked & $\mathrm{KCl}$ \\
S5 & 4 & 40 & Valinomycin & 5 & Unsoaked & $\mathrm{KCl}$ \\
S6 & 4 & 20 & Valinomycin & 5 & Unsoaked & $\mathrm{KCl}$ \\
S7 & 4 & 20 & Valinomycin & 5 & $0.1 \mathrm{M} \mathrm{KCl}$ & $\mathrm{KCl}$ \\
S8 & 4 & 20 & Valinomycin & 5 & $0.1 \mathrm{M} \mathrm{NaCl}$ & $\mathrm{KCl}$ \\
S9 & 1 & 40 & Valinomycin & 2 & Unsoaked & $\mathrm{NaCl}$ \\
S10 & 4 & 40 & Ionophore II & 5 & Unsoaked & $\mathrm{NaCl}$ \\
S11 & 4 & 40 & Ionophore III & 5 & Unsoaked & $\mathrm{NaCl}$ \\
\hline
\end{tabular}


Table 1 details all the different sensor designs that were tested. The data were analysed as described in Section 3.2 above and are plotted in Figure 6; a steeper gradient implies a more sensitive sensor.

It was determined from the results shown in Figure 6 a that the $5 \mathrm{wt} \%$ ionophore membrane was more sensitive; for cost-efficiency reasons, the ionophore concentration was not increased further, although this could lead to a higher sensitivity. Of the four different types of electrode (described in Section 2.2), Figure $6 \mathrm{~b}$ shows that $\mathrm{S} 6$ performs the best, which is unsurprising since it has the largest active area and path length.

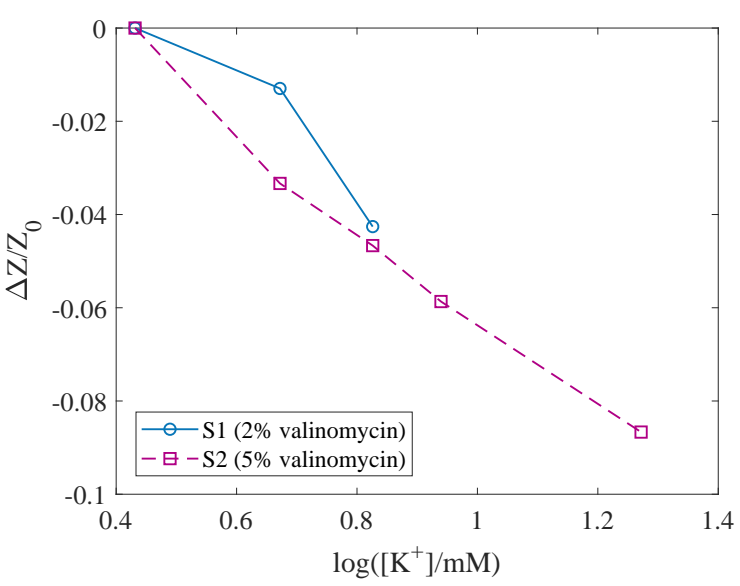

(a) Influence of valinomycin concentration on sensor response. Some of the higher $\mathrm{K}^{+}$concentration results for $\mathrm{S} 1$ are omitted, as they were inaccurate due to a hole in the membrane that formed during use.

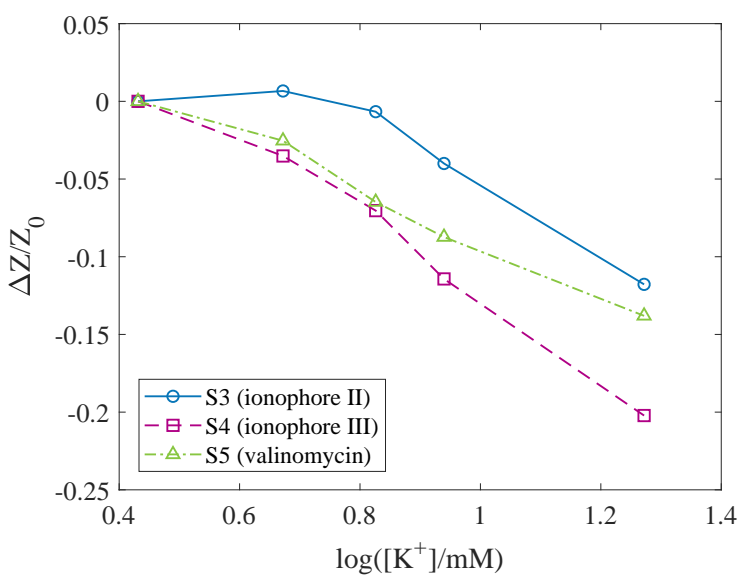

(c) Response of sensors with different ionophores.

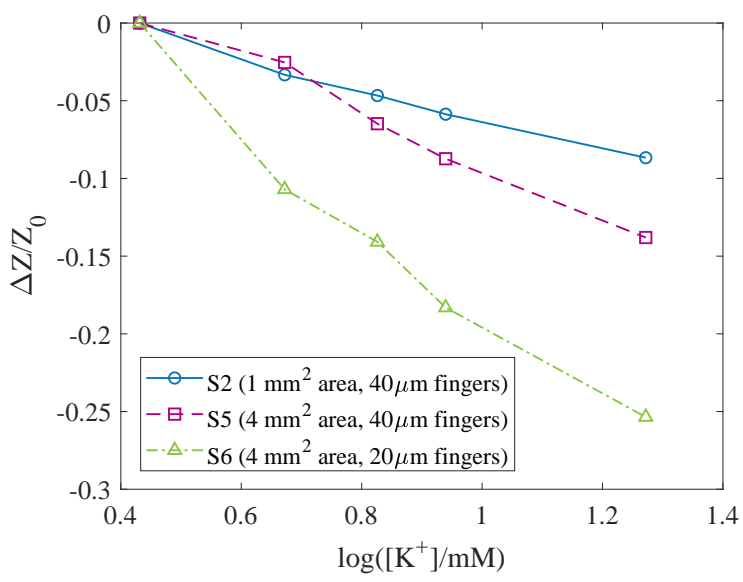

(b) Sensitivity of sensors with different electrode sizes. There were no good data obtained from a $1 \mathrm{~mm}^{2}$ area / $20 \mu \mathrm{m}$ finger width sensor, so this combination was not included here.

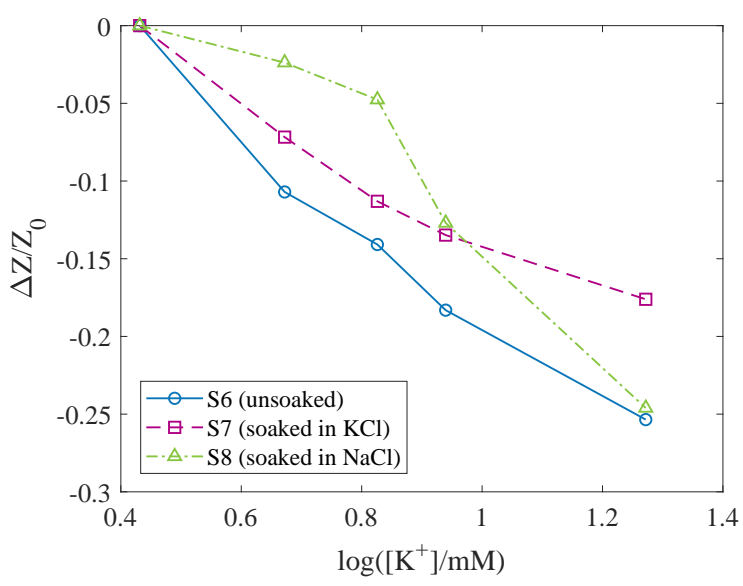

(d) Effect of soaking sensors before use.

Figure 6: Results of sensor optimisation.

Figure 6c shows clearly that ionophore II has the poorest response; ionophore III and valinomycin are comparable, with ionophore III perhaps responding slightly better. However, as described in Section 3.4 below, ionophore III shows a larger response to sodium than does valinomycin, which is undesirable.

Comparison of sensors S6, S7 and S8 (Figure 6d) shows that soaking in NaCl gives the poorest results, and leaving the membrane unsoaked is preferable to soaking in $\mathrm{KCl}$. On the other hand, the sensor that was soaked in $\mathrm{KCl}$ has a weaker time-dependence than the unsoaked one, which means that the extrapolation described in Section 3.2 is more reliable. For this reason, soaking could be preferable, although it also makes 
the fabrication process more complicated, so will be omitted for the sake of simplicity.

\subsection{Selectivity for potassium over sodium}

Sodium ions are a very common interference species for potassium-ion sensors, particularly in blood, as $\mathrm{Na}^{+}$is so much more abundant than $\mathrm{K}^{+}$; the $\mathrm{Na}^{+}: \mathrm{K}^{+}$ratio in a healthy person is typically around $35: 1$. Therefore, all three ionophores were tested for their $\mathrm{Na}^{+}$response, and this is plotted alongside their $\mathrm{K}^{+}$ response in Figure 7. It can clearly be seen that all three show a negligible response to sodium; any small deviations are most likely due to experimental error.

However, ionophore III appears to show a marginal negative $\mathrm{Na}^{+}$response. Although this could be experimental error, it is possible that it indicates that ionophore III complexes sodium as well as potassium. Since ionophore III has only a marginally better sensitivity than valinomycin, as seen in Figure 6c, valinomycin was determined to be the best ionophore overall. For a to-scale comparison of the $\mathrm{Na}^{+}$responses of each ionophore, see Figure S3 in the Supplementary Information.

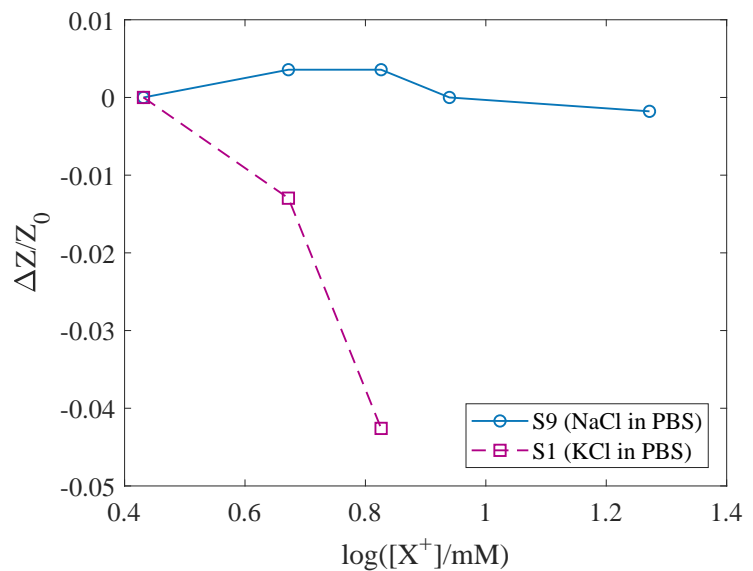

(a) Valinomycin sensor

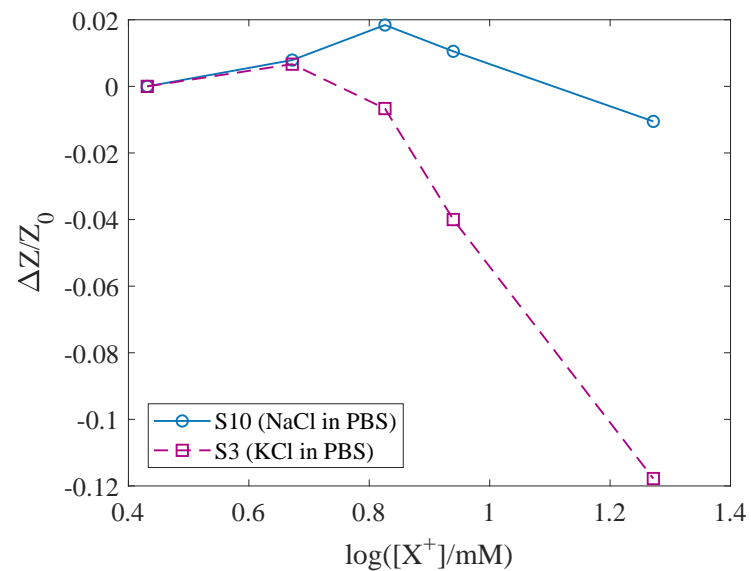

(b) Ionophore II sensor

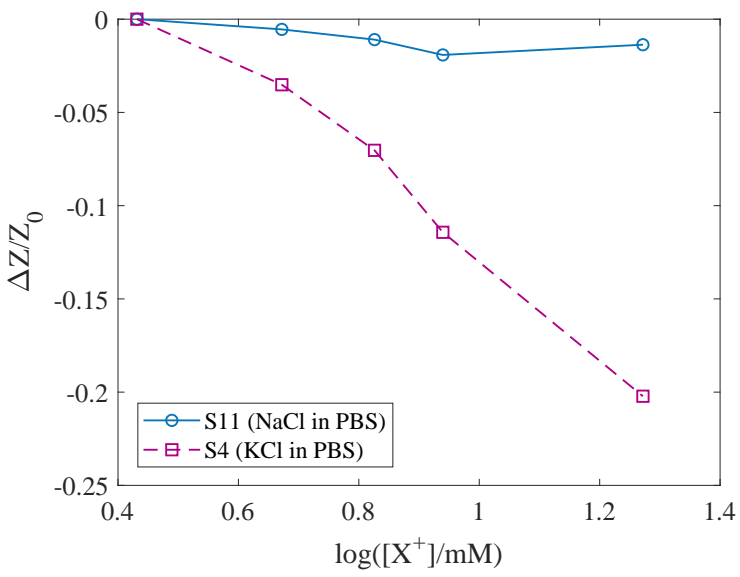

(c) Ionophore III sensor

Figure 7: Comparison of the responses to $\mathrm{K}^{+}$and $\mathrm{Na}^{+}$. In the $\mathrm{NaCl}$ experiments, $1 \mu \mathrm{L}$ aliquots of a $140 \mathrm{mM} \mathrm{NaCl}$ solution were added to the PBS solution to increase the $\mathrm{Na}^{+}$concentration in the cell by $2 \mathrm{mM}$ at a time, analogously to the process described in Section 2.4 for $\mathrm{KCl}$. On the x-axis, the Na ${ }^{+}$concentration has been scaled to aid comparison: $\left[\mathrm{X}^{+}\right]=\left[\mathrm{K}^{+}\right]=$ $\left(\left[\mathrm{Na}^{+}\right]-134.3 \mathrm{mM}\right)$. The base concentration of $\mathrm{NaCl}$ in PBS is $137 \mathrm{mM}$, compared to that of $\mathrm{KCl}$ which is $2.7 \mathrm{mM}$. 


\section{Conclusions}

In this work, a conductometric sensor was developed to detect potassium-ion concentration in a sample solution of the same concentration range as found in human blood. The potassium-selective membrane used in similar sensors in the past has been developed for ISEs; here, it was optimised specifically for use in conductometric sensors. It was found that a higher ionophore concentration of $5 \mathrm{wt} \%$ produced a better response than the $1-2 \mathrm{wt} \%$ used in most ISEs. Valinomycin was found to be the best overall ionophore for this application, and gold electrodes with the largest active area and narrowest finger width showed the best response of those tested.

Further membrane optimisation is needed to improve the sensitivity of the sensor and its response time. Calculation of the measurement accuracy possible within a certain time-frame is also necessary; for clinical use, a response time of a few minutes would be reasonable. This could be achieved by further studies and analysis of the time-dependent response, and by taking readings more frequently, so that curve-fitting could be done and a result obtained rapidly. However, the work appears very promising: individual sensors are small, compact and simple to produce, show a strong sensitivity to potassium ions and are not sensitive to sodium, and do not require a reference electrode.

\section{Funding}

This work was supported by Kidney Research UK [grant number RP_053_20160307]. C. Day is supported by a Vice-Chancellor's Award from the Cambridge Trust. T. Hutter is supported by the Henslow Fellowship at Darwin College, Cambridge, and L'Oréal UK \& Ireland Fellowship For Women In Science (2016). F. Karet Frankl is partly supported by the NIHR Cambridge Biomedical Research Centre.

The funding bodies had no involvement in study design; in the collection, analysis and interpretation of data; in the writing of the report; and in the decision to submit the article for publication.

Declarations of interest: none.

\section{References}

[1] J. Růžička, E. H. Hansen, E. A. Zagatto, Flow injection analysis: Part VII. Use of ion-selective electrodes for rapid analysis of soil extracts and blood serum. Determination of potassium, sodium and nitrate, Analytica Chimica Acta 88 (1) (1977) 1-16. doi:10.1016/S0003-2670(01)96043-X.

[2] S. G. Lemos, A. R. A. Nogueira, A. Torre-Neto, A. Parra, J. Artigas, J. Alonso, In-Soil Potassium Sensor System, J. Agric. Food Chem. 52 (19) (2004) 5810-5815. doi:10.1021/jf0492924.

[3] U. E. Spichiger, D. Freiner, E. Bakker, T. Rosatzin, W. Simon, Optodes in clinical chemistry: Potential and limitations, Sensors and Actuators B: Chemical 11 (1) (1993) 263-271. doi:10.1016/0925-4005(93)85264-B.

[4] L. van de Velde, E. d'Angremont, W. Olthuis, Solid contact potassium selective electrodes for biomedical applications a review, Talanta 160 (2016) 56-65. doi:10.1016/j.talanta.2016.06.050.

[5] A. Craggs, G. J. Moody, J. D. R. Thomas, PVC matrix membrane ion-selective electrodes. Construction and laboratory experiments, J. Chem. Educ. 51 (8) (1974) 541. doi:10.1021/ed051p541.

[6] R. D. Armstrong, G. Horvai, Properties of PVC based membranes used in ion-selective electrodes, Electrochimica Acta 35 (1) (1990) 1-7. doi:10.1016/0013-4686(90)85028-L.

[7] G. Horvai, E. Graf, K. Toth, E. Pungor, R. P. Buck, Plasticized poly(vinyl chloride) properties and characteristics of valinomycin electrodes. 1. High-frequency resistances and dielectric properties, Anal. Chem. 58 (13) (1986) 2735-2740. doi:10.1021/ac00126a034.

[8] K. N. Mikhelson, M. A. Peshkova, Advances and trends in ionophore-based chemical sensors, Russ. Chem. Rev. 84 (6) (2015) 555. doi:10.1070/RCR4506.

[9] Y. U. Budak, K. Huysal, M. Polat, Use of a blood gas analyzer and a laboratory autoanalyzer in routine practice to measure electrolytes in intensive care unit patients, BMC Anesthesiology 12 (2012) 17. doi:10.1186/1471-2253-12-17.

[10] J. Hu, A. Stein, P. Bühlmann, Rational design of all-solid-state ion-selective electrodes and reference electrodes, TrAC Trends in Analytical Chemistry 76 (2016) 102-114. doi:10.1016/j.trac.2015.11.004.

[11] A. Michalska, K. Maksymiuk, All-plastic, disposable, low detection limit ion-selective electrodes, Analytica Chimica Acta 523 (1) (2004) 97-105. doi:10.1016/j.aca.2004.07.020.

[12] H. Xu, X. Yang, Y. Wang, J. Zheng, Z. Luo, G. Li, Disposable blood potassium sensors based on screen-printed thick film electrodes, Measurement Science and Technology 21 (5) (2010) 055802. doi:10.1088/0957-0233/21/5/055802.

[13] M. Bamsey, A. Berinstain, M. Dixon, Development of a potassium-selective optode for hydroponic nutrient solution monitoring, Analytica Chimica Acta 737 (Supplement C) (2012) 72-82. doi:10.1016/j.aca.2012.05.024. 
[14] L. Xie, Y. Qin, H.-Y. Chen, Direct Fluorescent Measurement of Blood Potassium with Polymeric Optical Sensors Based on Upconverting Nanomaterials, Anal. Chem. 85 (5) (2013) 2617-2622. doi:10.1021/ac303709w.

[15] M. Tenjimbayashi, H. Komatsu, M. Akamatsu, W. Nakanishi, K. Suzuki, J. P. Hill, S. Shiratori, K. Ariga, Determination of blood potassium using a fouling-resistant PVDF-HFP-based optode, RSC Adv. 6 (17) (2016) 14261-14265. doi:10.1039/C5RA26514B.

[16] A. A. Shul'ga, B. Ahlers, K. Cammann, Ion-selective conductometric microsensors based on the phenomenon of specific salt extraction, Journal of Electroanalytical Chemistry 395 (1) (1995) 305-308. doi:10.1016/0022-0728(95)04205-3.

[17] K. Cammann, B. Ahlers, D. Henn, C. Dumschat, A. A. Shul'ga, New sensing principles for ion detection, Sensors and Actuators B: Chemical 35 (1) (1996) 26-31. doi:10.1016/S0925-4005(96)02008-4.

[18] U. Trebbe, M. Niggemann, K. Cammann, G. Fiaccabrino, M. Koudelka-Hep, S. Dzyadevich, O. Shulga, A new calciumsensor based on ion-selective conductometric microsensors - membranes and features, Fresenius J Anal Chem 371 (6) (2001) 734-739. doi:10.1007/s002160101093.

[19] G. R. Aitken, P. J. Roderick, S. Fraser, J. S. Mindell, D. O'Donoghue, J. Day, G. Moon, Change in prevalence of chronic kidney disease in England over time: Comparison of nationally representative cross-sectional surveys from 2003 to 2010 , BMJ Open 4 (9) (2014) e005480. doi:10.1136/bmjopen-2014-005480.

[20] M. Kerr, B. Bray, J. Medcalf, D. J. O'Donoghue, B. Matthews, Estimating the financial cost of chronic kidney disease to the NHS in England, Nephrol. Dial. Transplant. 27 Suppl 3 (2012) iii73-80. doi:10.1093/ndt/gfs269.

[21] J. S. You, Y. S. Park, H. S. Chung, H. S. Lee, Y. Joo, J. W. Park, S. P. Chung, S. H. Lee, H. S. Lee, Evaluating the Utility of Rapid Point-of-Care Potassium Testing for the Early Identification of Hyperkalemia in Patients with Chronic Kidney Disease in the Emergency Department, Yonsei Med J 55 (5) (2014) 1348-1353. doi:10.3349/ymj.2014.55.5.1348.

[22] A. E. Shvarev, D. A. Rantsan, K. N. Mikhelson, Potassium-selective conductometric sensor, Sensors and Actuators B: Chemical 76 (1-3) (2001) 500-505. doi:10.1016/S0925-4005(01)00601-3.

[23] M. Braiek, M. A. Djebbi, J.-F. Chateaux, N. Jaffrezic-Renault, A conductometric sensor for potassium detection in whole blood, Sensors and Actuators B: Chemical 235 (2016) 27-32. doi:10.1016/j.snb.2016.05.050. 